\title{
Should patients with papillary microcarcinoma undergo radioiodine ablation?
}

\author{
Leonard Wartofsky
}

Received: 31 May 2013/Accepted: 25 June 2013/Published online: 2 July 2013

(C) Springer Science+Business Media New York 2013

\section{Introduction}

Current management trends for patients with papillary thyroid microcarcinoma (PTMC) of $<1 \mathrm{~cm}$ are based upon a predicted excellent outcome without radioiodine ablation. Guidelines suggest that post-surgical ablation is not necessary for such small tumors and rather that the patients may be followed by monitoring serum thyroglobulin and performing periodic neck ultrasonography [1]. In this issue of Endocrine, Gallicchio et al. [2] report on a retrospective 14-month follow up of 85 pT1 patients with PTMC who did receive ablation of whom $35 \%$ were shown subsequently to have lymph node metastases on SPECT/CT. They suggest that perhaps we may have abandoned ablative therapy either prematurely or too broadly, and that the criteria for or against ablation of these PTMC patients need to be re-examined. Similar caution was raised recently by Malandrino et al. [3] from a survey of two cancer registries, one in Sicily and one in the USA, from which the same proportion, i.e., $35 \%$ of patients, were noted to have two or more risk factors for recurrence that included lymph nodes, multifocality, younger age, and extrathyroidal extension. The significance of lymph node metastases lies in their association with future recurrence. This issue was recognized in the most recent ATA Guidelines [1] which while not advocating radioiodine ablation for small tumors or even multifocality, did leave the door open for ablation of those tumors with higher risk features such as lymph node metastases, local invasiveness, or aggressive histologies such as tall cell, insular, or columnar variants of PTC.

L. Wartofsky $(\bowtie)$

Department of Medicine, Washington Hospital Center,

110 Irving Street, NW, Washington, DC 20010, USA

e-mail: leonard.wartofsky@medstar.net
It is conceivable that the selected cut-off size of $1 \mathrm{~cm}$ may be too arbitrary and can be further refined with better discrimination in regard to which PTMC's might be associated with lymph node metastases or subsequent recurrence. Thus, Lee et al. [4] proposed a tumor size of $7 \mathrm{~mm}$ as the cut off point for PTMC having noted that tumors $<7 \mathrm{~mm}$ were less likely to have aggressive features, such as lymph node metastases (30.6\%), compared to larger tumors of $7-10 \mathrm{~mm}$ of which $47.8 \%$ had central compartment lymph node metastases. Experience has taught us that the TNM pathologic classification of risk status is suboptimal or poorly applicable to many patients with thyroid cancer. Other recommendations for risk stratification that establish the risk of recurrence or establish subsequent risk based on responses to initial therapy are becoming more useful $[5,6]$, and could be considered in the postoperative decision for or against radioiodine ablation. Even when the preoperative diagnosis is uncomplicated PTMC, a decision might be made to recommend radioiodine ablation postoperatively on the basis of either the operative findings (e.g., incomplete tumor resection, aggressive histologic type, etc.) or a higher than expected serum thyroglobulin. Such patients would be re-stratified from "low risk" to "intermediate risk" for whom RAI ablation serves to facilitate disease surveillance and offers some benefit in improving the overall survival or reducing the risk of local relapse of disease.

What criteria or characteristics of PTMC might be useful as risk factors for recurrence? Male gender and lateral cervical node metastases were found by Kim et al. [7] to be associated with recurrence, and Zhang et al. [8] identified multifocality, male gender, tumor size $>6 \mathrm{~mm}$, and extrathyroidal extension as risk factors for lymph node metastasis. Their presence in patients with PTMC might warrant central neck lymph node dissection as well as 
subsequent radioiodine ablation. Opinions on this issue are polarized, and on the basis of the generally excellent clinical outcomes with the overwhelming majority of PTMC's, Pacini impugned the importance of risk factors including lymph node metastases as having no major impact on outcome even when identified [9]. I would caution against overly generalized recommendations in clinical medicine, even though the latter opinion is likely to be correct in the majority of cases. Rather, I believe that we must develop better approaches to a clinically based differentiation of those PTMC's that will exhibit biologically benign behavior from those that will not.

In the very near future, it is likely that we will be looking to some aspect of molecular mutational analysis of these tumors to determine which mutations in a microcarcinoma are associated with extrathyroidal extension, multifocality, or lateral neck lymph node metastases [9, 10]. The group of Nikiforov et al. [11] proposed risk stratification of patients with PTMC based upon their BRAF status , and several studies [12, 13], but not all [14, 15] associate the presence of BRAF with more aggressive biological behavior. Clearly, all PTMC's will not exhibit the same behavior, and we are likely to have only "scratched the surface" in the discovery and understanding of other genomic mutations that impact biological behavior and clinical outcomes. While the analysis of Gallicchio et al. implies some benefit of radioiodine ablation for PTMC, we should remain mindful that there are pitfalls inherent in the potential adverse effects of overly aggressive radioisotope therapy [10]. As in most things, a balanced approach is required and we await further clarification by mutational analysis of a rational basis for decision making in the management of PTMC.

\section{REFERENCES}

1. D.S. Cooper, G.M. Doherty, B.R. Haugen, R.T. Kloos, S.L. Lee, S.J. Mandel, E.L. Mazzaferri, B. McIver, F. Pacini, M. Schlumberger, S.I. Sherman, D.L. Steward, R.M. Tuttle, Revised American thyroid association management guidelines for patients with thyroid nodules and differentiated thyroid cancer. Thyroid 19, 1167-1214 (2009)

2. R. Gallicchio, S. Giacomobono, D. Capacchione, A. Nardelli, F. Barbato, Nappia, T. Pellegrino, G. Storto, Should patients with remnants from thyroid microcarcinoma really not be treated with Iodine-131 ablation? Endocrine 30(2), 506 (2013)

3. P. Malandrino, G. Pellegriti, M. Attard, M.A. Violi, C. Giordano, L. Sciacca, C. Regalbuto, S. Squatrito, R. Vigneri, Papillary thyroid microcarcinomas: a comparative study of the characteristics and risk factors at presentation in two cancer registries. J. Clin. Endocrinol. Metab. 98, 1427-1434 (2013)

4. K.J. Lee, Y.J. Cho, S.J. Kim, S.C. Lee, J.G. Kim, C.J. Ahn, D.H. Lee, Analysis of the clinicopathologic features of papillary thyroid microcarcinoma based on 7-mm tumor size. World J. Surg. 35, 318-323 (2011)

5. R.M. Tuttle, G. Rondeau, N.Y. Lee, A risk-adapted approach to the use of radioactive iodine and external beam radiation in the treatment of well-differentiated thyroid cancer. Cancer Control 18, 89-95 (2011)

6. M.G. Castagna, F. Maino, C. Cipri, Belardini V. Theodoropoulou, A. Cevenini, G. Pacini F, Delayed risk stratification to include the response to initial treatment (surgery and radioiodine ablation) has better outcome predictivity in differentiated thyroid cancer patients. Eur. J. Endocrinol. 165, 441-446 (2011)

7. T.Y. Kim, S.J. Hong, J.M. Kim, W.G. Kim, G. Gong, J.S. Ryu, W.B. Kim, S.-C. Yun, Y.K. Shong, Prognostic parameters for recurrence of papillary thyroid microcarcinoma. BMC Cancer $\mathbf{8}$, 296 (2008). doi:10.11186/1471-2407-8-296

8. L. Zhang, W.-J. Wei, Q.-H. Ji, Y.-X. Zhu, Z.-Y. Wang, C.-P. Huang, J.Q. Shen, D.-S. Li, Y. Wu, Risk factors for neck lymph node metastasis in papillary thyroid microcarcinoma: a study of 1066 patients. J. Clin. Endocrinol. Metab. 97, 1250-1257 (2012)

9. F. Pacini, Management of papillary microcarcinoma: primum non nocere! J. Clin. Endocrinol. Metab. 98, 1391-1393 (2013)

10. L. Wartofsky, Management of papillary microcarcinoma: primum non nocere? J. Clin. Endocrinol. Metab. 97, 1169-1172 (2012)

11. L.A. Niemeier, A.H. Kuffner, C. Song, S.E. Carty, S.P. Hodak, L. Yip, R.L. Ferris, G.C. Tseng, R.R. Seethala, S.O. Lebeau, M.T. Stang, C. Coyne, J.T. Johnson, A.F. Stewart, Y.E. Nikiforov, A combined molecular-pathologic score improves risk stratification of thyroid papillary microcarcinoma. Cancer 118, 2069-2077 (2011)

12. M. Xing, A.S. Alzahrani, K.A. Carson, D. Viola, R. Elisei, B. Bendlova, L. Yip, C. Mian, F. Vianello, R.M. Tuttle, E. Robenshtok, J.A. Fagin, E. Puxeddu, L. Fugazzola, A. Czarniecka, B. Jarzab, C.J. O’Neill, M.S. Sywak, A.K. Lam, G. Riesco-Eizaguirre, P. Santisteban, H. Nakayama, R.P. Tufano, S.I. Pai, M.A. Zeiger, W.H. Westra, D.P. Clark, R. Clifton-Bligh, D. Sidransky, P.W. Ladenson, V. Sykorova, Association between BRAF V600E mutation and mortality in patients with papillary thyroid cancer. JAMA 309(14), 1493-1501 (2013)

13. R. Elisei, D. Viola, L. Torregrossa, V. Belardini, A. Theodoropoulou, G. Cevenini, F. Pacini, The BRAF V600E mutation is an independent, poor prognostic factor for the outcome of patients with low-risk intrathyroid papillary thyroid carcinoma: singleinstitution results from a large cohort study. J. Clin. Endocrinol. Metab. 97, 4390-4398 (2012)

14. S.T. Lee, S.W. Kim, C.S. Ki, J.H. Jang, J.H. Shin, Y.L. Oh, J.W. Kim, J.H. Chung, Clinical implications of highly sensitive detection of the BRAF V600E mutation in fine needle aspirations of thyroid nodules: a comparative analysis of three molecular assays in 4585 consecutive cases in a BRAF V600E mutationprevalent area. J. Clin. Endocrinol. Metab. 97, 2299-2306 (2012)

15. G. Gandolfi, V. Sancisi, F. Torricelli, M. Ragazzi, A. Frasoldati, S. Piana, A. Ciarrocchi, Allele percentage of the BRAF V600E mutation in papillary thyroid carcinoma and corresponding lymph node metastases: no evidence for a role in tumor progression. J. Clin. Endocrinol. Metab. 98, E934-E942 (2013) 